\title{
SOME NEW ITERATED HARDY-TYPE
}

INEQUALITIES AND APPLICATIONS

\section{A. L. Bernardis AND PEDro ORTega Salvador}

Abstract. We characterize some iterated Hardy inequalities previously studied by R. Oinarov and A. Kalybay and, as an application, we give new simpler proofs of the characterizations of the weighted inequalities for the bilinear Hardy operator.

Mathematics subject classification (2010): 26D10, 26D15, 42B99.

Keywords and phrases: Bilinear operators, Hardy type operators, weighted inequalities, weights.

\section{REFERENCES}

[1] M. I. Aguilar Cañestro, P. Ortega Salvador and C. Ramírez Torreblanca, Weighted bilinear Hardy inequalities, J. Math. Anal. Appl. 387 (2012), no. 1, 320-334.

[2] J. BRadley, Hardy inequalities with mixed norms, Canad. Math. Bull. 21 (1978), no. 4, 405-408.

[3] A. Gogatishvili and L. PICK, Discretization and anti-discretization of rearrangement-invariant norms, Publ. Mat. 47 (2003), 311-358.

[4] A. Kufner And L. E. Persson, Weighted inequalities of Hardy type, World Scientific, 2003.

[5] Q. LAI, Weighted modular inequalities for Hardy type operators, Proc. London Math. Soc. 79 (1999), 649-672.

[6] V. G. MAZ’ JA, Sobolev Spaces, Springer-Verlag, 1985.

[7] B. Muckenhoupt, Hardy's inequality with weights, Studia Math. 44 (1972), 31-38.

[8] R. Oinarov and A. Kalybay, Three-parameter weighted Hardy type inequalities, Banach J. Math. Anal. 2 (2008), no. 2, 85-93.

[9] R. Oinarov And A. Kalybay, Weighted inequalities for a class of semiadditive operators, Ann. Funct. Anal. 6 (2015), no. 4, 155-171. 